\title{
Image Recovering for Sparse-aperture Systems
}

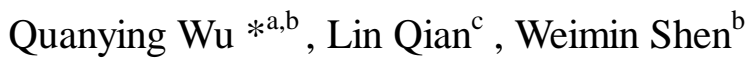 \\ ${ }^{a}$ University of Science and Technology of Suzhou, Suzhou 215009, China \\ ${ }^{\mathrm{b}}$ Institute of Modern Optical Technology, Soochow University, Suzhou 215006, China \\ ${ }^{\mathrm{c}}$ School of Phys Sci\&Tech, Soochow University, Suzhou 215006, China
}

\begin{abstract}
Sparse-aperture imaging systems are desirable for aerospace applications because they can capture the same resolution as a filled aperture while reducing the systems' size and weight. A novel sparse-aperture model named dual three-sub-aperture is proposed. By comparing with the famous Golay 6, dual three-sub-aperture is regarded as a better configuration for aerospace remote sensing. But the images of sparse-aperture systems become blurry because of the modulation transfer function (MTF) loss. It is necessary to optimize the image quality by image restoration process. In order to achieve ideal images, image filter technique has been studied. First, the imaging simulations of dual three-sub-aperture system and the Golay 6 with different fill factor are generated. The images formed by these systems are recovered by means of proper filters. Then different kinds of noises and different noise levels are added, various filters with different parameters are applied to recover these images. And the optimal deblurred images are gained. Through the quantitative evaluations of its image quality it is shown that the mentioned filter technique can be used to effectively improve the quality of the images degraded by the MTF's loss, i.e. the details in images can be enhanced and its edges be sharpened.
\end{abstract}

Keywords: space remote sensing, sparse-aperture, image quality, filters, image recovery

\section{INTRODUCTION}

In space-borne systems for surveillance applications, it is very important to achieve relatively high resolution. Higher resolution can be obtained with a larger collecting area. But the system's weight with a larger collecting area becomes heavy. Such systems are not suited to aerospace remote sensing because of developing larger and larger boosters capable of handling the mass and volume of the larger filled-aperture systems. A single large monolithic telescope for an imaging satellite becomes prohibitive to launch into orbit due to the size and weight of the primary mirror, and its cost is expensive. A sparse-aperture imaging system can capture the same resolution as a filled-aperture while reducing the systems' size and weight. But the images of sparse-aperture systems become blurry because of the modulation transfer function (MTF) loss and the decreasing of the signal-to-noise ratio (SNR), therefore, image processing is needed to gain a high resolution image.

In this paper, we have proposed a novel sparse-aperture model named dual three-sub-aperture, shown in Fig.1. There are nine sub-apertures which diameters are $d$, each three sub-apertures make up of a sub-pupil which diameter is $D_{1}$, three sub-pupils make up of a main pupil which diameter is D.

The image simulations for dual three-sub-aperture and the Golay $6^{1,2,3}$ (shown in Fig.2) with different fill factors are generated, and different kinds of noises and noise levels are added. The main tasks are to recover these images' quality of sparse-aperture by filters. The quantitative evaluations of image quality and the edges of images are given. By comparing with the famous Golay 6 sparse-aperture system, dual three-sub-aperture is regarded as a better configuration for aerospace remote sensing. 


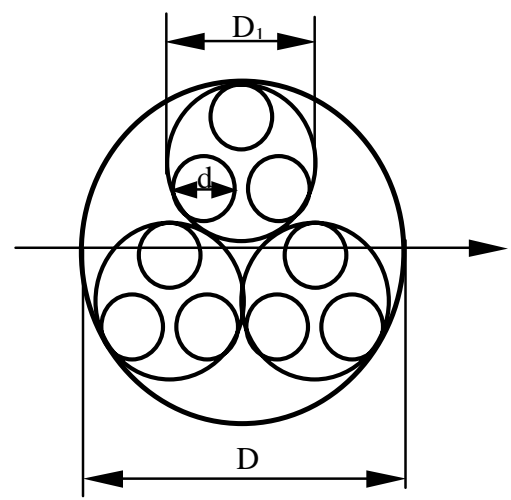

Fig.1. Configuration of dual three-sub-aperture

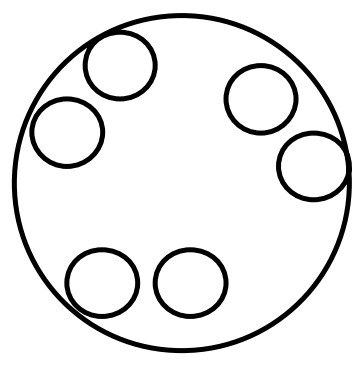

Fig.2. Configuration of the Golay6

\section{MTF OF DUAL THREE-SUB-APERTURE AND THE GOLAY 6}

\subsection{Three models and MTF of dual three- sub-aperture}

There are three kinds of configurations of dual three-sub-aperture, as Model I, Model II and Model III, shown in Fig.3-5. In Model I, three sub-pupils are tangent each other. In Model II, three sub-apertures are tangent each other. In Model III, neither three sub-pupils nor three sub-apertures are tangent.

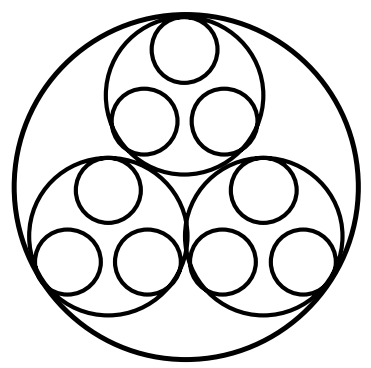

Fig.3. Model I

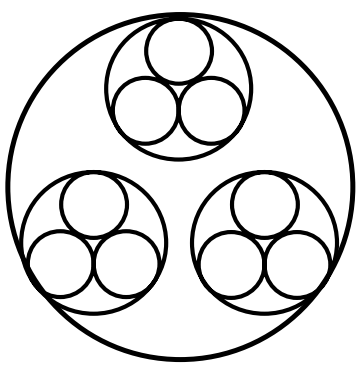

Fig.4. Model II

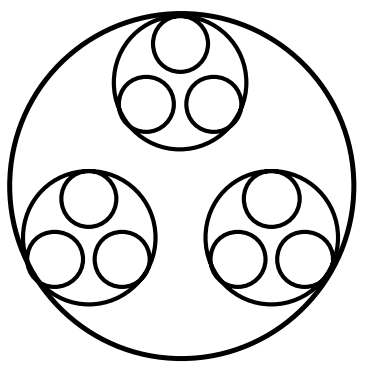

Fig.5. Model III

The analytical formula of MTF is as follows:

$$
\begin{gathered}
M T F=M T F_{d}+\frac{1}{9} M T F_{d} * \sum_{k} \sum_{l} c_{k} \delta\left(u-\rho_{k} \cos \alpha_{k l}, v-\rho_{k} \sin \alpha_{k l}\right) \\
k=1,2 \ldots 8, l=1,2 \cdots 6
\end{gathered}
$$

$\rho_{k}$ and $\alpha_{k l}$ are determined by the locative position between nine sub-apertures. 


$$
\begin{cases}\left.\rho_{1,2}=r_{g}^{2}+r_{s}^{2}-2 r_{g} r_{s} \cos \left(\frac{2}{3} \pi+\theta_{0}\right)\right)^{\frac{1}{2}} / \lambda f^{\prime} & \alpha_{k l}=(2 l-1) \frac{\pi}{6}+(-1)_{k} \theta_{0}, \\ \rho_{3,4}=\sqrt{3} r_{s} / \lambda f^{\prime} & \Delta \alpha=3 r_{g} / 2 \rho_{1,2} \\ \rho_{5}=\sqrt{3} r_{g} / \lambda f^{\prime} & \alpha_{k l}=l \times \frac{\pi}{3}+(-1)^{k} \theta_{0} \\ \rho_{6,7,8}=2 r_{s} \sin \left[(k-6) \frac{\pi}{3}+\theta_{0}\right] / \lambda f^{\prime} & \alpha_{k l}=l \times \frac{\pi}{3}\end{cases}
$$

The MTF of sub-aperture of dual three-sub-aperture is given by

$$
\begin{array}{rlr}
M T F_{d} & = \begin{cases}\frac{2}{\pi}\left(\cos ^{-1} \frac{\rho}{\rho_{c}}-\frac{\rho}{\rho_{c}}\left[1-\left(\frac{\rho}{\rho_{c}}\right)^{2}\right]^{\frac{1}{2}}\right. & 0 \leq \rho \leq \rho_{c} \\
0 & \rho \geq \rho_{c}\end{cases} \\
\rho_{c} & =d / \lambda f^{\prime}
\end{array}
$$

Three dimensional MTF of Model I which fill factor is $20 \%$ are shown in Fig.6, Three models for dual three-sub-aperture, with images of their MTF which fill factor is $20 \%$ are shown in Fig.7.

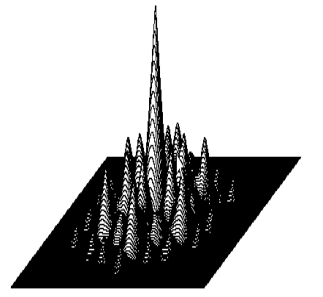

Fig.6. Three dimensional $\mathrm{MTF}(\mathrm{F}=20 \%)$
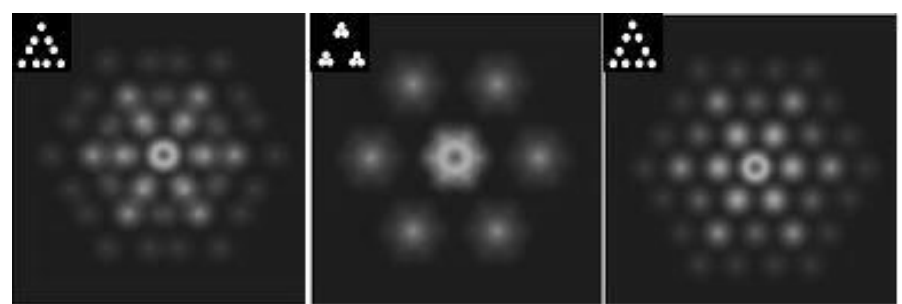

Fig.7. Three models for dual three-sub-aperture, with images of their MTF

\subsection{MTF comparison between dual three sub-aperture and the Golay 6}

Fig. 8 shows circled aperture, the Golay 6 ,and the dual three sub-aperture with a $20 \%$ fill factor, together with images of corresponding aperture MTFs.
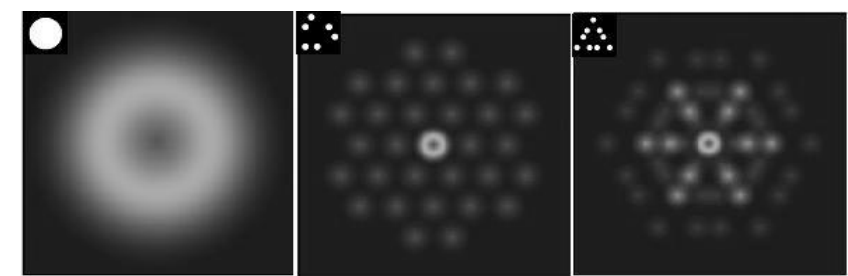

Fig.8. Three apertures, with images of their corresponding MTFs

Fig. 9 and Fig.10 show the MTF curves of dual three-sub-aperture and the Golay 6 with different fill factors at the direction of maximal MTF and minimal MTF. From the curves we can see that the MTF increases as the fill factor increases. The MTF of sparse aperture decreases at most spatial frequencies, but the spatial-frequency cutoff does not change, the highest spatial frequency that can be imaged does not change. The MTFs of dual three-sub-aperture corresponding to the normalized frequency of approximate 0.25 to 0.35 at the maximal direction are better than those of 
the Golay 6.
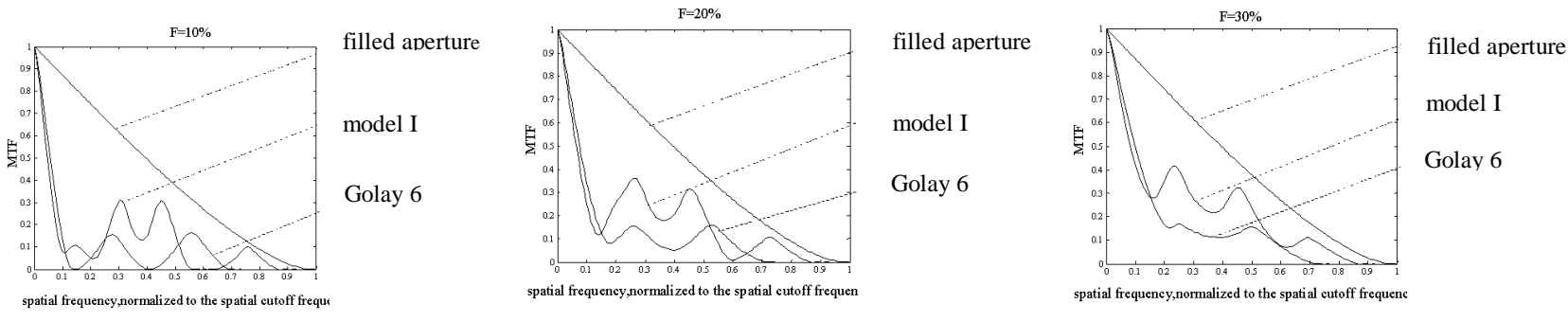

Fig.9. The MTF curves of dual three-sub-aperture and the Golay 6 at the direction of maximal MTF
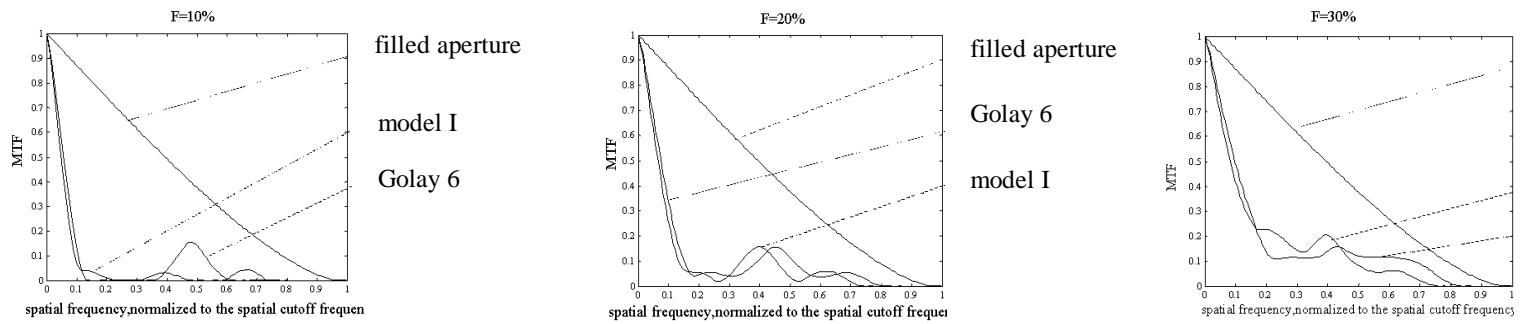

filled aperture

model I

Golay 6

Fig.10. The MTF curves of dual three-sub-aperture and the Golay 6 at the direction of minimal MTF

\section{IMAGE SIMULATIONS}

In order to understand the image quality of sparse-aperture systems, different fill factor sparse-aperture systems of dual three-sub-aperture and the Golay 6 are modeled, and images are simulated. A resolution board (shown in Fig.10-a) is used as an object, imaged by a paraxial optical system $(\mathrm{D} / \mathrm{f}=1 / 20)$. Fig. 10 -b shows a diffraction limited image.

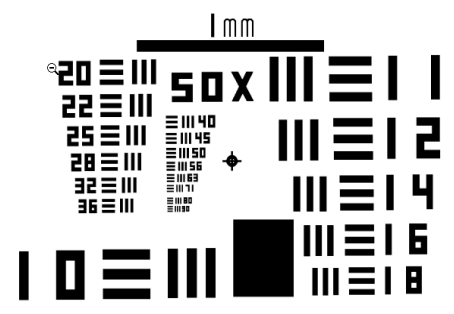

Fig. 10 -a Object

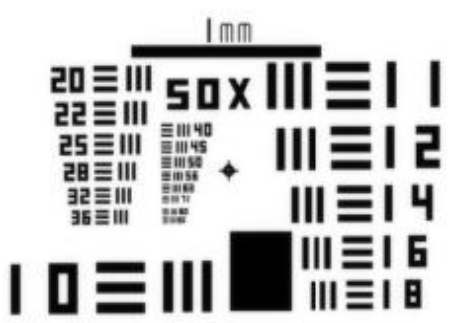

Fig.10 -b Diffraction limited image
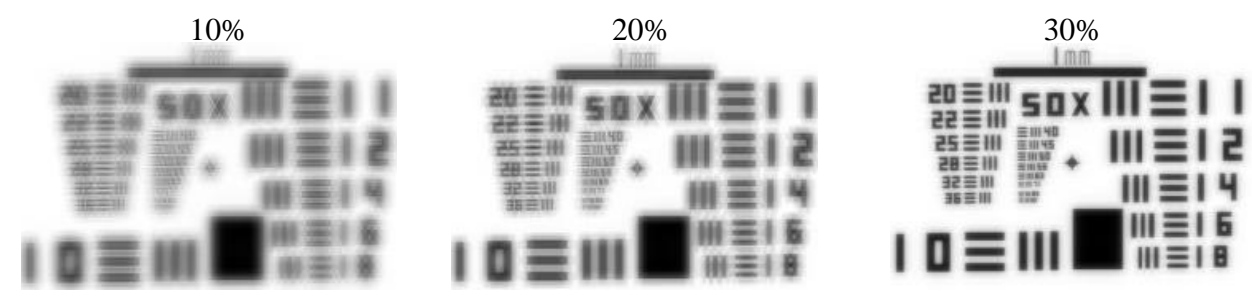

Fig.11 Image simulations for the dual three- sub-aperture with increasing fill factor 

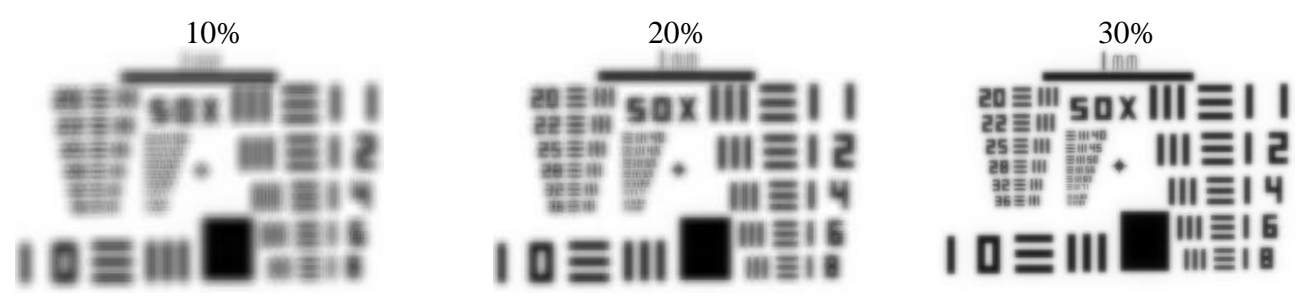

Fig.12 Image simulations for the Golay 6 sparse aperture with increasing fill factor

The image simulations are shown for dual three-sub-aperture and the Golay 6 sparse aperture in Fig.11.and Fig.12. It is apparent from the image simulations that the image quality varies dramatically for the different sparse apertures as the fill factor increases. The image becomes clearer as increasing fill factor. It should be noted that if the fill factor is low enough to produce zeros within the optical bandpass of the MTF, then the image quality becomes rather bad.

\section{IMAGE RECOVERING WITH FILTERS}

\subsection{Wiener filter}

The images for sparse-aperture become blurry because of the loss of the MTF, hence an image restoration process is necessary to recover the sharpness. Wiener filter is applied to recover the images, but we find that the recovered images are not ideal, then the modified Wiener filter has been shown to restore the images for sparse-aperture systems.

A image form ${ }^{4}$ is:

$$
w(x, y)=f(x, y) * h(x, y)+n(x, y)
$$

where $w(x, y)$ denotes the image of sparse-aperture system, $f(x, y)$ is the origin object, $h(x, y)$ is the PSF of sparse-aperture, $n(x, y)$ is the additive noise, the symbol * denotes convolution.

From convolution theory, Eq.(3) can be expressed in the frequency domain as:

$$
W(u, v)=F(u, v) \cdot H(u, v)+N(u, v)
$$

The objective is to find an estimate $\hat{f}(x, y)$ of the sparse-aperture so that the mean square error between $\hat{f}(x, y)$ and $f(x, y)$ is minimized. This mean square error is given by:

$$
e^{2}=\int_{-\infty}^{+\infty} \int_{-\infty}^{+\infty}|f(x, y)-\hat{f}(x, y)|^{2} d x d y
$$

It is assumed that the signal and noise are uncorrelated, that the noise has zero mean, and that the gray levels in the estimate are linearly related to the levels in the degraded image. Based on these conditions, the estimative spectrum with the general Wiener filters is given by:

$$
\hat{F}(u, v)=\left[\frac{H^{*}(u, v)}{|H(u, v)|^{2}+K}\right] W(u, v)
$$

where $H^{*}(u, v)$ denotes the complex conjugate of $H(u, v),|H(u, v)|^{2}=H^{*}(u, v) H(u, v)$, 
$K=\frac{P_{n}(u, v)}{P_{f}(u, v)} \begin{aligned} & , \mathrm{K} \text { is the ratio of the noise power spectrum and the origin signal power spectrum. In practice, } \mathrm{K} \text { is not } \\ & \text { kut we find that the recovered images are unacceptable. The modified Wiener filter has been studied } \\ & \text { to improve the image quality. }\end{aligned}$

\subsection{Modified Wiener filter}

The term in square bracket of Eq.(6) is defined as Wiener filter, we contrive to find a function to replace Wiener filter. This filter is defined as modified Wiener filter ${ }^{5}$. We have chosen a Gaussian function to multiply by the term in square bracket. The Gaussian function is given as:

$$
t_{p s f}(x, y)=e^{-\left(x^{2}+y^{2}\right) / 2 \sigma^{2}}
$$

where $\sigma$ is a positive real constant for adjusting the width of Gaussian span. Incorporating Eq.(7) into (6) yields:

$$
\hat{F}(u, v)=\left[\frac{H^{*}(u, v) T_{p s f}(u, v)}{|H(u, v)|^{2}+K}\right] W(u, v)
$$

where $T_{p s f}(u, v)$ is the Fourier transform of $t_{p s f}(x, y)$. The term in square bracket of Eq.(8) is defined as the modified Wiener filter.

\subsection{Image recovering}

Images for dual three sub-aperture and the Golay 6 sparse aperture with different kinds of noises and different SNR levels are simulated. The images are also restored by modified Wiener filters.

4.3.1. Images and image recovering with different kinds of noises for dual three sub-aperture and the Golay 6 Image quality varies as the kinds of noises and SNR levels are changed. Different noises are added in the images of the sparse aperture. Three noise-added figures for dual three sub-aperture and the Golay 6 with 20\%-fill-factor are shown in Fig.13. and Fig.14. The noise level is $10 \%$, and the noise kinds are random noise, gaussian noise and salt\&pepper noise, respectively.
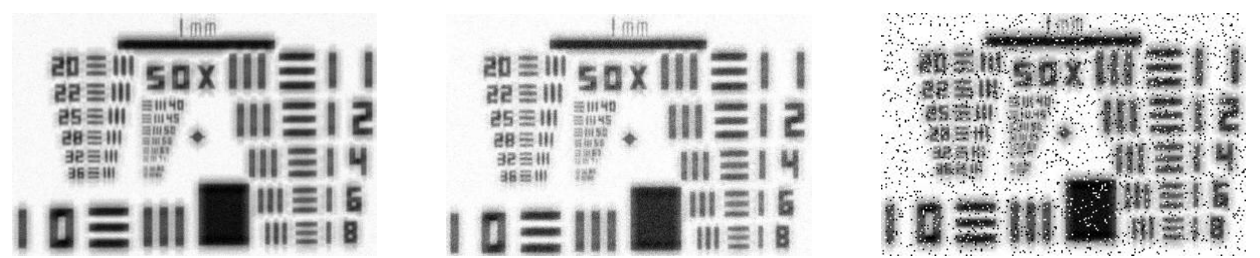

Fig.13. Noise-added images for dual three-sub-aperture
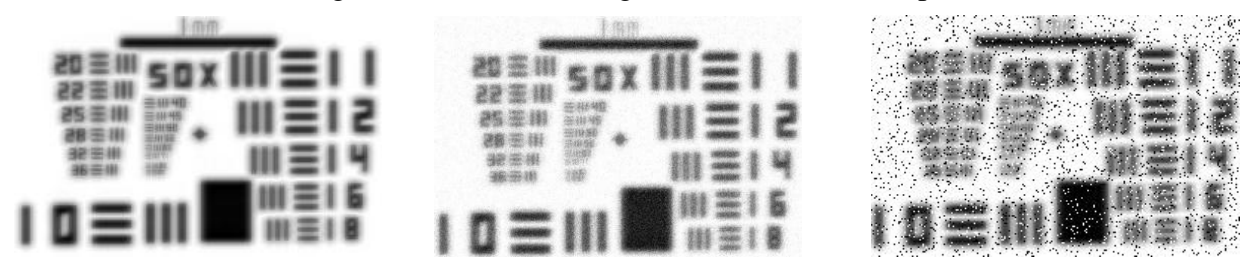

Fig.14. Noise-added images for the Golay 6 sparse system

We can see from Fig.13. and Fig.14. that the image quality becomes more indistinct than before. It is necessary to restore 
these images. Each image is processed with modified Wiener filter to enhance the detail in the images. The optimal filter for each image was determined by processing each image with a series of filters with varying $\mathrm{K}$ and $\sigma$, then, the optimal values of $\mathrm{K}$ and $\sigma$ in Eq.(8) are chosen to gain ideal images. The restored images of modified Wiener filter are shown in Fig.15.
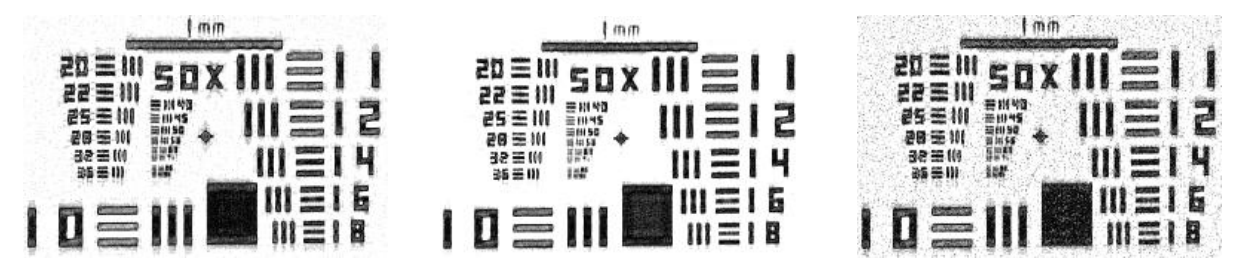

Fig.15-a. The restored images with different noises for dual three-sub-aperture
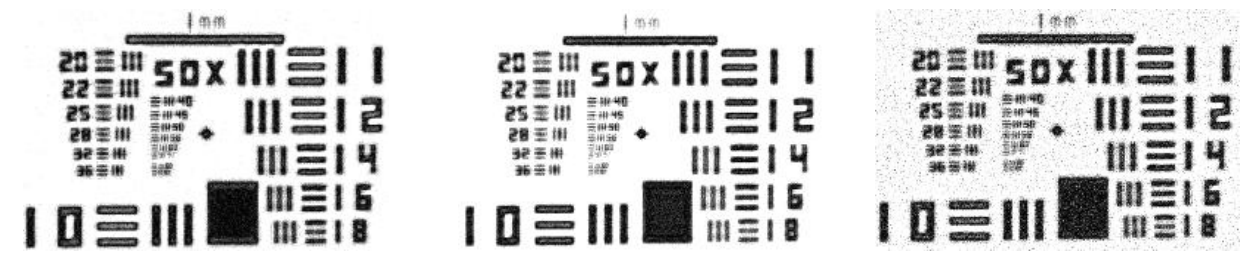

Fig.15-b. The restored images with different noises for the Golay 6

\subsubsection{Images and image recovering with different noise levels for dual three sub-aperture and Golay 6}

When the noise level is rather large, the image quality becomes extremely bad. We show the noise-added images which noise kind is salt\&pepper and noise level is 20\%. Fig.16-a and Fig.16-b show the images that are noise-added and are filtered for dual three sub-aperture and the Golay 6 . Although the noise levels change, the added-noise images blur, the deblurring effects in these images are ideal. It is apparent that the restored image for dual three sub-aperture looks better than ones for the Golay 6.
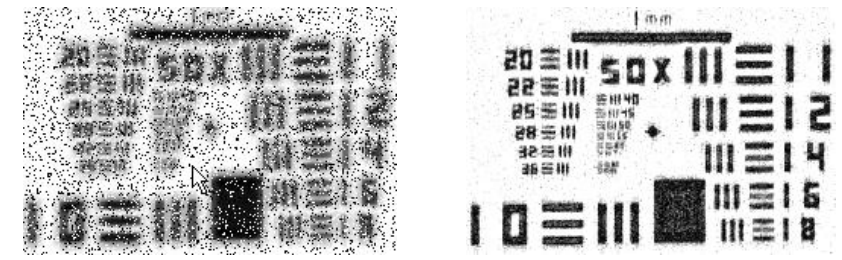

Fig.16-a. The images of added-noise and restored for dual three-sub-aperture with the $20 \%$-fill- factor
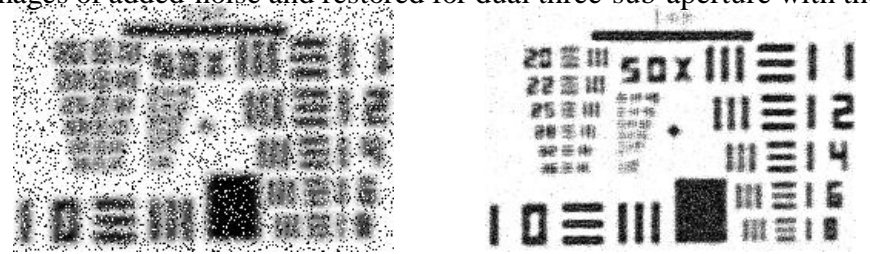

Fig.16-b. The images of added-noise and restored for the Golay 6 aperture system with the $20 \%$-fill-factor

\section{THE EVALUATION OF IMAGES QUALITY AND SHARPNESS OF IMAGES}

\subsection{Standard deviation of the sparse aperture}

For comparison of images quality between images of the sparse aperture systems and $100 \%$-fill-factor systems, we did a calculation of standard deviation (std) ${ }^{6}$. 


$$
\operatorname{std}(i)=\sqrt{\frac{\sum\left(E_{i}-E_{0}\right)^{2}}{m \times n-1}}
$$

where $E_{i}$ is the intensity of gray scale levels of the sparse aperture, $E_{0}$ is the intensity of gray scale levels of the $100 \%$-fill-factor system. $\quad m \times n$ is the pixel size of metric. Results of calculation are shown in Table 1

Table 1 Standard deviation (std) of the sparse aperture systems

\begin{tabular}{|c|c|c|c|c|}
\hline Image & & fill factor $10 \%$ & fill factor 20\% & fill factor 30\% \\
\hline \multirow{2}{*}{ Dual three- sub-aperture } & added-noise & 03534 & 0.3289 & 0.3251 \\
\cline { 2 - 5 } & filtered & 0.3042 & 0.3105 & 0.2877 \\
\hline \multirow{2}{*}{ Golay 6 } & added-noise & 0.3601 & 0.3316 & 0.3316 \\
\cline { 2 - 5 } & filtered & 0.3147 & 0.3146 & 0.2942 \\
\hline
\end{tabular}

From Table 1, we can see that the standard deviation (std) of the sparse aperture systems decreases as the fill factor increases. It shows that the image quality becomes better with increasing the fill factor. After filtered, the image quality is improved. The image simulations generated and the evaluation of image quality show that dual three sub-aperture is slightly better than the Golay 6.

5.2 Edges sharpness of dual three-sub-aperture

Image edges are unclear by sparse aperture imaging. We find that the image edges become sharp after filtering, shown in Fig. 17.

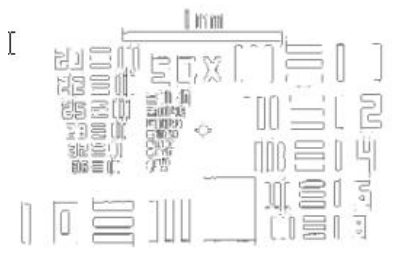

Fig.17-a. Image edges before added-noise

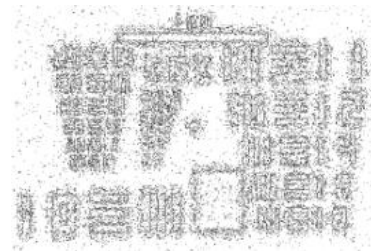

Fig.17-b. Image edges after added-noise

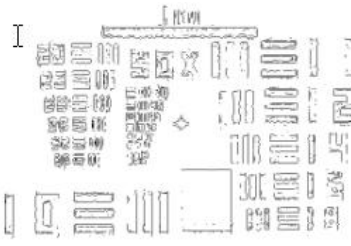

Fig.17-c. Image edges after filtered

\section{CONCLUSION}

A novel model named dual three-sub-aperture is proposed. Its MTF and three configurations are generated. The comparisons between dual three-sub-aperture and the Golay 6 sparse aperture are given. Wiener filter and modified Wiener filter are discussed. The image simulations generated with dual three-sub-aperture and the Golay 6 sparse aperture show that image quality decreases as the fill factor decreases. Image quality with the same fill factor varies because of different kinds of noises and different noise levels. Wiener filter can be used to restore the degraded images, but the filter parameter should be chosen so that the optimal filtered images are gained. By images processing with Wiener filter, the image edges are sharpened. The standard deviations of the sparse aperture images are calculated to show the improvement of image quality in each situation. 


\section{ACKNOWLEDGMENTS}

We would like to thank our advisor Mingqiu Xue for his guide. He gives us a lot of help in our research work. His challenges to the team is crucial to spurring it on to approach the problems from a new point of view each time the path ahead appeared to be blocked. The work on this topic is sponsored by JiangSu' Natural Science Fund of China under grant No.BK2004202. It is also supported by Fund of JiangSu' Key Lab of Modern Optical Technology, Soochow University.

\section{REFERENCES}

1. Robert D. F., Theodore A T, "Image Quality of Sparse-aperture Designs for Remote Sensing[J]", Optical Engineering, 41 (8), $1957 \sim 1968,2002$.

2. Meinel A. B., Meinel P M, "Large Sparse-Aperture Space Optical Systems[J]",Optical Engineering, 41 (8),1983 $\sim 1994,2002$.

3. James E. E., Richard A. R., "Performance Characteristics of Phased Array and Thinned Aperture Optical Telescopes[J]", Optical Engineering, 27 (9), $762 \sim 768,1988$.

4. Castleman K. R., Digital Image Processing [M], 218 229, Prentice-Hall International Inc, 1998.

5. Yufeng Zheng, Xiaohui Cui, Mark P. Wachowiak, and Adel S.Elmaghraby, "CT scout Z-resolution improvement with image restoration methods", Proceedings of SPIE, vol.5032(2003),1851 1857.

6. Ling Guan, Rabab K.Ward, "Restoration of stochastically blurred images by the geometrical mean filter", Optical Engineering, 29 (4), $289 \sim 295,1990$.

*wqycyh@tom.com;phone 8651265113796; fax 8651265112232 